\title{
A NOTE ON THE MULTIPLICATION FORMULAS FOR THE BERNOULLI AND EULER POLYNOMIALS
}

\section{CARLITZ}

1. Polynomials of order 1 . The following three formulas are well known $[4$, pp. 18,24$]$ :

$$
\begin{aligned}
& B_{m}(k x)=k^{m-1} \sum_{s=0}^{k-1} B_{m}\left(x+\frac{s}{k}\right), \\
& E_{m}(k x)=k^{m} \sum_{s=0}^{k-1}(-1)^{s} E_{m}\left(x+\frac{s}{k}\right) \quad \text { (k odd), } \\
& E_{m-1}(k x)=-\frac{2 k^{m-1}}{m} \sum_{s=0}^{k-1}(-1)^{8} B_{m}\left(x+\frac{s}{k}\right) \quad(k \text { even }),
\end{aligned}
$$

where $B_{m}(x), E_{m}(x)$ denote the polynomials of Bernoulli and Euler in the usual notation. It is perhaps not so familiar that (1.1) and (1.2) characterize the polynomials. More precisely, as Nielsen has pointed out $[3$, p. 54], if a normalized polynomial satisfies (1.1) for a single value $k>1$, then it is identical with $B_{m}(x)$; similarly if a normalized polynomial satisfies (1.2) for a single odd $k>1$, then it is identical with $E_{m}(x)$. For some generalizations see [1].

The situation for (1.3) is clearly different. For consider the equation

$$
g_{m-1}(k x)=-\frac{2 k^{m-1}}{m} \sum_{8=0}^{k-1}(-1)^{s} f_{m}\left(x+\frac{s}{k}\right) \quad(k \text { even }),
$$

where $f_{m}(x), g_{m-1}(x)$ denote normalized polynomials of degree $m$ and $m-1$, respectively. (That the multiplier $-2 k^{m-1} / m$ is necessary can be seen by examining the coefficient of $x^{m-1}$ in both members of (1.4).) Now if $k$ is a fixed even integer $\geqq 2$ and $f_{m}(x)$ is an arbitrary normalized polynomial of degree $m$, then (1.4) determines $g_{m-1}(x)$ as a normalized polynomial of degree $m-1$; hence (1.4) for a single value of $k$ does not suffice to determine both $f_{m}(x)$ and $g_{m-1}(x)$. However, as we shall see, if (1.4) holds for two distinct values of $k$, then $f_{m}(x)$ and $g_{m-1}(x)$ are uniquely determined-except for an additive constant in $f_{m}(x)$.

Put

Presented to the Society, September 5, 1952; received by the editors August 4, 1952. 


$$
\begin{array}{rlr}
f_{m}(x) & =\sum_{r=0}^{m}\left(\begin{array}{c}
m \\
r
\end{array}\right) a_{m r} B_{r}(x) & \left(a_{m m}=1\right) \\
g_{m-1}(x) & =\sum_{r=0}^{m-1}\left(\begin{array}{c}
m-1 \\
r
\end{array}\right) b_{m-1, r} E_{r}(x) & \left(b_{m-1, m-1}=1\right)
\end{array}
$$

Substituting in (1.4) gives

$$
\begin{aligned}
\sum_{r=0}^{m-1}\left(\begin{array}{c}
m-1 \\
r
\end{array}\right) b_{m-1, r} E_{r}(k x) & \\
= & -\frac{2 k^{m-1}}{m} \sum_{r=1}^{m}\left(\begin{array}{c}
m \\
r
\end{array}\right) a_{m r} \sum_{s=0}^{k-1}(-1)^{s} B_{r}\left(x+\frac{s}{k}\right) \\
& =\frac{k^{m-1}}{m} \sum_{r=0}^{m}\left(\begin{array}{c}
m \\
r
\end{array}\right) \frac{r}{k^{r-1}} E_{r-1}(k x),
\end{aligned}
$$

by virtue of (1.3). Comparing coefficients of $E_{r}(k x)$, we get

$$
b_{m-1, r-1}=k^{m-r} a_{m r} \quad(r \geqq 1) .
$$

If we now assume that (1.4) holds also for the value $h \neq k$, then (1.6) implies $b_{m-1, r-1}=h^{m-r} a_{m r}(r \geqq 1)$, from which it is evident that

$$
b_{m-1, r-1}=a_{m r}=0 \quad(1 \leqq r<m) .
$$

Thus (1.5) becomes

$$
f_{m}(x)=B_{m}(x)+a_{m 0}, \quad g_{m-1}(x)=E_{m-1}(x),
$$

where $a_{m 0}$ is an arbitrary constant. This proves the following

THEOREM 1. If $f_{m}(x), g_{m-1}(x)$ denote normalized polynomials of degree $m$ and $m-1$ respectively, which satisfy (1.4) for two distinct values of $k$, then (1.7) holds; in other words, except for an additive constant, $f_{m}(x)$ is identical with $B_{m}(x)$ and $g_{m-1}(x)$ is identical with $E_{m-1}(x)$.

If in addition we assume $f_{m}(0)=B_{m}$, then it is clear that $f_{m}(x)$ $=B_{m}(x)$.

It may be of interest to remark that if we assume $f_{m}^{\prime}(x)=m f_{m-1}(x)$, so that $\left\{f_{m}(x)\right\}$ form an Appell set, then (1.5) becomes

$$
\begin{aligned}
f_{m}(x) & =\sum_{r=0}^{m}\left(\begin{array}{c}
m \\
r
\end{array}\right) a_{m-r} B_{r}(x)=(B(x)+a)^{m}, \\
g_{m-1}(x) & =\sum_{r=0}^{m-1}\left(\begin{array}{c}
m-1 \\
r
\end{array}\right) k^{m-1-r} a_{m-1-r} E_{r}(x)=(E(x)+k a)^{m-1} .
\end{aligned}
$$

In particular then $\left\{g_{m}(x)\right\}$ also form an Appell set. (Note that in 
deriving (1.8) we have assumed that (1.4) holds for a single value of k.)

If we also suppose that

$$
f_{m}(1-x)=(-1)^{m} f_{m}(x)
$$

then (1.8) becomes

$$
(-1)^{m} f_{m}(x)=\sum_{r=0}^{m}(-1)^{r}\left(\begin{array}{c}
m \\
r
\end{array}\right) a_{m-r} B_{r}(x),
$$

since $B_{m}(x)$ satisfies (1.9). It follows that $a_{m-r}=0$ for $m-r$ odd.

It may be of interest to add a word concerning the equation

$$
f_{m-1}(k x)=-\frac{2 k^{m-1}}{m} \sum_{s=0}^{k-1}(-1)^{\circ} f_{m}\left(x+\frac{s}{k}\right) \quad(k \text { even }) .
$$

In the first place if (1.10) is satisfied for two distinct values of $k$, then it follows from (1.7) that

$$
f_{m}(x)=B_{m}(x)+a_{m 0}=E_{m}(x),
$$

but it is easily seen that (1.11) is impossible for $m>2$. If then we assume that (1.10) holds for a single value of $k$, we have

$$
f_{m-1}(k x+1)+f_{m-1}(k x)=\left(2 k^{m} / m\right)\left(f_{m}(x+1)-f_{m}(x)\right) .
$$

Thus if $f_{m-1}(x)$ is assigned, $f_{m}(x)$ is determined except for an additive constant. If we make the additional assumption that $\left\{f_{m}(x)\right\}$ is an Appell set, then (1.8) applies and we get the condition

$$
\frac{t}{2} \frac{e^{t}+1}{e^{t}-1} \sum_{0}^{\infty} \frac{a_{m} t^{m}}{m !}=\sum_{0}^{\infty} \frac{a_{m} k^{m} t^{m}}{m !},
$$

which is equivalent to

$$
k^{m} a_{m}=\sum_{r} a_{m-2 r} B_{2 r}
$$

By means of (1.12) the $a_{m}$ are uniquely defined; in particular $a_{2 r+1}=0$.

2. Polynomials of higher order. If we define $B_{m}^{(n)}(x), E_{m}^{(n)}(x)$ by means of

$$
\begin{aligned}
& \left(\frac{t}{e^{t}-1}\right)^{n} e^{x t}=\sum_{m=0}^{\infty} B_{m}^{(n)}(x) \frac{t^{m}}{m !} \\
& \left(\frac{2}{e^{t}+1}\right)^{n} e^{x t}=\sum_{m=0}^{\infty} E_{m}^{(n)}(x) \frac{t^{m}}{m !}
\end{aligned}
$$


then (1.1), (1.2), (1.3) are easily extended to the general case [4, Chapter 6$]$. We shall be concerned only with the generalized version of (1.3), namely

$$
\begin{aligned}
& \sum_{s=0}^{n(k-1)}(-1)^{\prime} C_{s} B_{m}^{(n)}\left(x+\frac{s}{k}\right) \\
& =\frac{m(m-1) \cdots(m-n+1)}{(-2)^{n} k^{m-n}} E_{m-n}^{(n)}(k x) \quad(k \text { even, } n \geqq 1),
\end{aligned}
$$

where $C_{8}$ is the coefficient of $x^{*}$ in the expansion of $(1+x+\cdots$ $\left.+x^{k-1}\right)^{n}$; note that $C_{8} \neq 0$. As above we consider the equation

$$
\begin{aligned}
\sum_{s=0}^{n(k-1)}(-1)^{\circledR} C_{s} f_{m}\left(x+\frac{s}{k}\right) & \\
& =\frac{m(m-1) \cdots(m-n+1)}{(-2)^{n} k^{m-n}} g_{m-n}(x) \quad(k \text { even }),
\end{aligned}
$$

where $f_{m}(x)$ and $g_{m-n}(x)$ are normalized polynomials. Now put

$$
\begin{aligned}
f_{m}(x) & =\sum_{r=0}^{m}\left(\begin{array}{c}
m \\
r
\end{array}\right) a_{m r} B_{r}^{(n)}(x) & \left(a_{m m}=1\right), \\
g_{m-n}(x) & =\sum_{r=0}^{m-n}\left(\begin{array}{c}
m-n \\
r
\end{array}\right) b_{m r} E_{r}^{(n)}(x) & \left(b_{m-n, m-n}=1\right) .
\end{aligned}
$$

Then substitution in (2.3) leads without difficulty to

$$
b_{m-n, r-n}=k^{m-r} a_{m r} \quad(r \geqq n),
$$

which is a direct generalization of (1.6). Comparison with the proof of Theorem 1 leads to

TheOREM 2. Let $m \geqq n$. If $f_{m}(x)$ and $g_{m-n}(x)$ denote normalized polynomials of degree $m$ and $m-n$, respectively, which satisfy (2.3) for two distinct values of $k$, then $g_{m-n}(x)=E_{m-n}^{(n)}(x)$ and $f_{m}(x)=B_{m}^{(n)}(x)+p_{n-1}(x)$, where $p_{n-1}(x)$ denotes an arbitrary polynomial of degree $\leqq n-1$.

We remark that the discussion following Theorem 1 can readily be extended to the general case $n \geqq 1$.

3. Eulerian polynomials. Put

$$
\frac{1-\alpha}{1-\alpha e^{t}} e^{x t}=\sum_{m=0}^{\infty} \frac{\phi_{m}(x, \alpha)}{m !} t^{m} \quad(\alpha \neq 1)
$$

then $\phi_{m}(x, \alpha)$ is a normalized polynomial of degree $m$ in $x$ and may be 
called an Eulerian polynomial (with parameter $\alpha$ ). In particular we note that

$$
\phi_{m}(x,-1)=E_{m}(x),
$$

since for $\alpha=-1$ the left member of (3.1) reduces to the generating function for $E_{m}(x)$.

By means of (3.1) it is easy to verify the formula (compare $[2, p$. $825]$ )

$$
\phi_{m-1}(k x, \rho)=\frac{(\rho-1) k^{m-1}}{m} \sum_{r=0}^{k-1} \rho^{r} B_{m}\left(x+\frac{r}{k}\right),
$$

where $\rho^{k}=1, \rho \neq 1$; for $\rho=-1, k$ even, it is clear from (3.2) that (3.3) reduces to (1.3). We therefore consider the equation

$$
g_{m-1}(k x)=\frac{(\rho-1) k^{m-1}}{m} \sum_{r=0}^{k-1} \rho^{r} f_{m}\left(x+\frac{r}{k}\right),
$$

where $\rho$ is a fixed primitive $l$ th root of unity, $l>1, k \equiv 0(\bmod l)$, and $f_{m}(x)$ and $g_{m-1}(x)$ are normalized in the usual way. Then if we put $f_{m}(x)=\sum_{r=0}^{m}\left(\begin{array}{c}m \\ r\end{array}\right) a_{m r} B_{r}(x), \quad g_{m-1}(x)=\sum_{r=0}^{m-1}\left(\begin{array}{c}m-1 \\ r\end{array}\right) b_{m-1, r} \phi_{r}(x, \rho)$,

we find that (3.4) and (3.3) imply $b_{m-1, r-1}=k^{m-r} a_{m r}(r \geqq 1)$. Then exactly as in the proof of Theorem 1 we get the following

Theorem 3. Let $\rho$ denote a fixed primitive lth root of unity, $l>1$. If $f_{m}(x)$ and $g_{m-1}(x)$ denote normalized polynomials of degree $m$ and $m-1$, respectively, which satisfy (3.4) for two distinct values of $k$ $\equiv 0(\bmod l)$, then

$$
f_{m}(x)=B_{m}(x)+a_{m 0}, \quad g_{m-1}(x)=\phi_{m-1}(x, \rho),
$$

where $a_{m 0}$ is an arbitrary constant.

Once again the discussion following Theorem 1 can be extended to polynomials satisfying (3.4).

\section{REFERENCES}

1. L. Carlitz, The multiplication formulas for the Bernoulli and Euler polynomials, Mathematics Magazine (to appear).

2. G. Frobenius, Über die Bernoulli'schen Zahlen und die Euler'schen Polynome, Preuss. Akad. Wiss. Sitzungsber. (1910) pp. 809-847.

3. N. Nielsen, Traitê elémentaire des nombres de Bernoulli, Paris, 1923.

4. N. E. Nörlund, Vorlesungen über Differenzenrechnung, Berlin, 1924.

DUKE UNIVERSITY 